\title{
Negative Thermal Expansion in Nanostructured Intermediate-Valence $\mathrm{YbAl}_{3}$
}

\author{
Cristina Echevarria-Bonet ${ }^{1}{ }^{(0)}$, Maria de la Fuente Rodriguez ${ }^{2}$, Jose I. Espeso ${ }^{2}$, \\ Jesus Angel Blanco Rodriguez ${ }^{1}$, Inés Puente Orench ${ }^{3,4}$, Daniel P. Rojas ${ }^{5}$, \\ Lidia Rodríguez Fernández ${ }^{6}$, Francois Fauth ${ }^{7}$, and Luis Fernández Barquín ${ }^{2}$ \\ ${ }^{1}$ Department of Physics, University of Oviedo, 33007 Oviedo, Spain \\ ${ }^{2}$ Departamento de Ciencias de la Tierra y Física de la Materia Condensada, Facultad de Ciencias, Universidad de Cantabria, 39005 \\ Santander, Spain \\ ${ }^{3}$ Institut Laue-Langevin, 38042 Grenoble, France \\ ${ }^{4}$ Instituto de Ciencia de Materiales de Aragón, Spanish National Research Council-Universidad de Zaragoza, 50009 Zaragoza, Spain \\ ${ }^{5}$ Departamento de Estructuras y Física de Edificación, Escuela Técnica Superior de Arquitectura de Madrid, Universidad Politécnica de \\ Madrid, 28040 Madrid, Spain \\ ${ }^{6}$ Servicio de Microscopía Electrónica de Transmisión, Universidad de Cantabria, 39005 Santander, Spain \\ ${ }^{7}$ CELLS—ALBA Synchrotron, E-08290 Barcelona, Spain
}

Received 31 Jul 2019, revised 2 Sep 2019, accepted 9 Sep 2019, published 18 Sep 2019, current version 29 Oct 2019.

Abstract-Interest in the strongly correlated electron system $\mathrm{YbAl}_{3}$ has been recently renewed by the observation of a breakdown of coherence effects and Fermi liquid behavior when the alloy's particle size is reduced to around $11 \mathrm{~nm}$. Powder diffraction measurements using neutron and synchrotron radiation allow us to estimate the thermal expansion of the nanostructured $\mathrm{YbAl}_{3}$. A region of negative thermal expansion in the nanostructured material occurs at higher temperatures compared to that of bulk $\mathrm{YbAl}_{3}$. This shows that the reduction in the size of $\mathrm{YbAl}_{3}$ not only affects electronic properties, but also the lattice dynamics of this material.

Index Terms—Nanomagnetics, intermediate valence, thermal expansion, neutron diffraction, synchrotron X-ray diffraction.

\section{INTRODUCTION}

The strongly correlated electron system (SCES) $\mathrm{YbAl}_{3}$ alloy has attracted much attention in the last decades due to the fact that it exhibits a great variety of cutting-edge physical phenomena, such as intermediate valence (IV), Kondo resonance, and Fermi-liquid (FL) behavior [van Daal 1974]. All these behaviors are related to the hybridization of the $4 \mathrm{f}$ state with the conduction band electrons. Among the great variety of experiments performed in $\mathrm{YbAl}_{3}$, we could cite magnetic [Hiess 2000] or thermoelectric [Rowe 2002] measurements and the effect of the hydrostatic pressure [Ohara 2010] or the magnetic field (and disorder) [Ebihara 2003], procuring a great deal of complementary information on the just related attractive phenomena. Valence fluctuations occur in both bulk [Suga 2005] and nanostructured alloys [Rojas 2008], leading to changes in the Fermi surface topology (Lifshiftz transition) of the Kondo lattice IV $\mathrm{YbAl}_{3}$ [Chatterjee 2017].

In bulk form, this alloy crystallizes in the cubic $\mathrm{AuCu}_{3}$-type structure (Pm-3m), with a cell parameter $a=4.2036$ (3) $\AA$ [Moriarty 1966]. Temperature-dependent magnetic susceptibility has been found to have a broad maximum at around $125 \mathrm{~K}$ and to follow a Curie-Weiss law with an effective moment $4.2 \mu_{B}$ [Bauer 2004]. The well-known maximum in the magnetic susceptibility appears in IV compounds and is consistent with a high Kondo temperature $T_{K} \approx 600-700 \mathrm{~K}$

International Conference on Fine Particle Magnetism, Gijón, Spain, 26-31 May 2019.

Corresponding author: Cristina Echevarria-Bonet (e-mail: echevarriacristina@ uniovi.es).

Digital Object Identifier 10.1109/LMAG.2019.2942262
[Bud'ko 2008]. Below $40 \mathrm{~K}$, an FL behavior ( $T^{2}$ dependence of the electrical resistivity) is connected to a coherence temperature of $40 \mathrm{~K}$ [Bauer 2004].

A change in the Kondo lattice behavior of bulk $\mathrm{YbAl}_{3}$ takes place when the alloy is in the nanoparticle state $(\approx 12 \mathrm{~nm})$. Measurements of the electrical resistivity show inhibited coherence effects and deviation from the standard Fermi liquid behavior ( $T^{2}$ dependence). These results are interpreted as being due to the effect of the disruption of the periodicity of the array of Kondo ions provoked by the size reduction process [Echevarria-Bonet 2018]. Coherence effects in bulk $\mathrm{YbAl}_{3}$ [Bauer 2004] are modified in nanostructures of this alloy [EchevarriaBonet 2018]. In this sense, $\mathrm{Yb}_{1-x} \mathrm{Lu}_{x} \mathrm{Al}_{3}$ series of bulk alloys have been also studied, and changes in specific heat, susceptibility, and electrical resistivity were found, suggesting that coherence effects are very sensitive to lattice order [Ebihara 2003].

Moreover, negative thermal expansion (NTE) occurs in bulk $\mathrm{YbAl}_{3}$ at low temperatures $(T<20 \mathrm{~K})$, whereas for the diluted alloy $\mathrm{Yb}_{0.1} \mathrm{Lu}_{0.9} \mathrm{Al}_{3}$, this region of NTE is not present [Bud'ko 2008], relating the presence of an Yb IV state to the existence of NTE. This behavior, or that of an almost zero thermal expansion, is not restricted to IV systems only. The classic example is the well-known $\mathrm{Fe}_{0.64} \mathrm{Ni}_{0.36}$, Invar alloy [Guillaume 1897], but there are other simpler systems, such as $\mathrm{Au}$ [Li 2002] or $\mathrm{CuO}$ [Zheng 2008], which have been reported to present NTE as nanoparticles but not in bulk, in different temperatures ranges [Attfield 2018]. NTE has also been measured in systems, such as the frustrated magnetic insulator $\mathrm{CdCr}_{2} \mathrm{O}_{4}$ [Rossi 2019], rare-earth intermetallic alloys $\mathrm{R}_{2} \mathrm{Fe}_{17}(\mathrm{R}=$ rare earth) [Alvarez-Alonso 2011], or the permanent magnet family $\mathrm{R}_{2} \mathrm{Fe}_{14} \mathrm{~B}$ [Buschow 1987]. 


\section{EXPERIMENTAL TECHNIQUES}

A starting polycrystalline $\mathrm{YbAl}_{3}$ pellet was prepared by arc melting of suitable amounts of pure constituents $\mathrm{Yb}(3 \mathrm{~N}), \mathrm{Al}(5 \mathrm{~N})$ (Alfa) under protective Ar atmosphere. It was subsequently annealed at $750{ }^{\circ} \mathrm{C}$ for five days in vacuum $\left(\sim 10^{-3} \mathrm{mbar}\right)$ in order to improve crystallinity and ensure homogenization of samples. The reduction in the particle size of bulk $\mathrm{YbAl}_{3}$ was achieved by mechanical milling, which is considered a practical route to obtain large quantities of nanocrystalline materials. Amounts around $4 \mathrm{~g}$ of bulk $\mathrm{YbAl}_{3}$ were crushed and then milled in a planetary high-energy ball milling system (Retsch PM 400/2) at a rotation speed of $200 \mathrm{r} / \mathrm{min}$. The initial powders were placed in tungsten carbide containers using a ball/sample weight ratio of 12:1. These containers were hermetically closed in a glove box in an Ar (99.99\%) atmosphere to minimize powder oxidation. The milling procedure was carried out following successive steps of $5 \mathrm{~min}$ of clockwise and anticlockwise rotations with a 5 min intermediate stop. Material was collected at $70 \mathrm{~h}$ of milling time. In order to avoid oxidation, handling and storage were carried out in a glove box under Ar atmosphere.

Synchrotron radiation powder X-ray diffraction (SR-PXD) patterns were acquired at room temperature $(\mathbf{R T})$ in the Materials Science and Powder Diffraction (MSPD) beamline at ALBA Synchrotron (wavelength, $\lambda=0.4126 \AA$ ). Powder neutron diffraction (ND) experiments were carried out in the diffractometer D1B, ILL (France) using a wavelength $\lambda=2.52 \AA$. A temperature-dependent study was performed from 2 to $300 \mathrm{~K}$.

SR-PXD and ND patterns were refined by the Rietveld method (through the FULLPROF Suite [Rodriguez-Carvajal 1993]) including resolution function calibration standards, thus taking into account the instrumental broadening. The fit was carried out using a ThompsonCox-Hastings function, allowing the calculation of the particle size $(D)$ and strain $(\eta)$.

Transmission electron microscopy (TEM) was performed in a Jeol 2100 microscope in order to check the nanocrystalline arrangement.

DC magnetization measurements were performed in a Quantum Design Physical Property Measurement System, in a standard zerofield-cooled protocol, with $\mu_{0} \mathrm{H}=1 \mathrm{~T}$ in the temperature range 2$300 \mathrm{~K}$.

\section{RESULTS}

\section{A. Structural Characterization}

The analysis of the X-ray diffraction pattern of the bulk alloy is consistent with a cubic crystal structure of the $\mathrm{AuCu}_{3}$-type (spacegroup Pm-3m) with a refined unit-cell parameter $a=4.2023(1) \AA$, in good agreement with previously reported data [Moriarty 1966]. A small percentage of $\mathrm{YbAl}_{2}$ is also found in the patterns. This fact has been reported previously [Görlach 2005], and subsequent mechanical milling results in helping reaction to finish and $\mathrm{YbAl}_{3}$ is formed [Echevarria-Bonet 2014].

Fig. 1 shows the SR-PXD patterns of the $70 \mathrm{~h}$ milled $\mathrm{YbAl}_{3}$ alloy (hereinafter $70 \mathrm{~h}-\mathrm{YbAl}_{3}$ ) at RT. Rietveld refinements provided the same structure than in the bulk (cubic, Pm-3m) with a lattice parameter $a$ $=4.2076(1) \AA$. Particle size was decreased down to $D=11(1) \mathrm{nm}$ and strain increased up to $\eta=0.40(1) \%$. The refinements led to

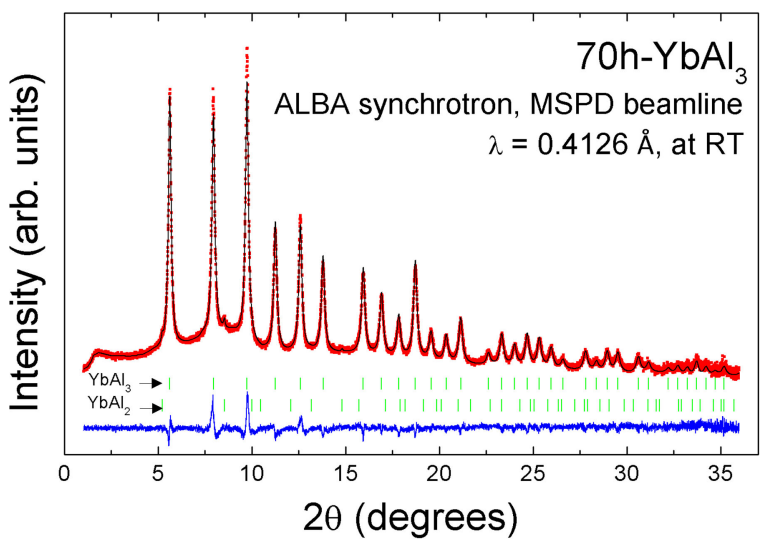

Fig. 1. Rietveld refinement of the SR-PXD pattern for $70 \mathrm{~h}-\mathrm{YbAl}_{3}$ at $\mathrm{RT}$. The theoretical calculation is depicted as a continuous (black) line and the experimental data as (red) points. The blue line below the spectra is the difference between the calculated and experimental patterns. Bragg reflections of the $\mathrm{YbAl}_{3}$ and $\mathrm{YbAl}_{2}$ phases are also depicted as green vertical lines.

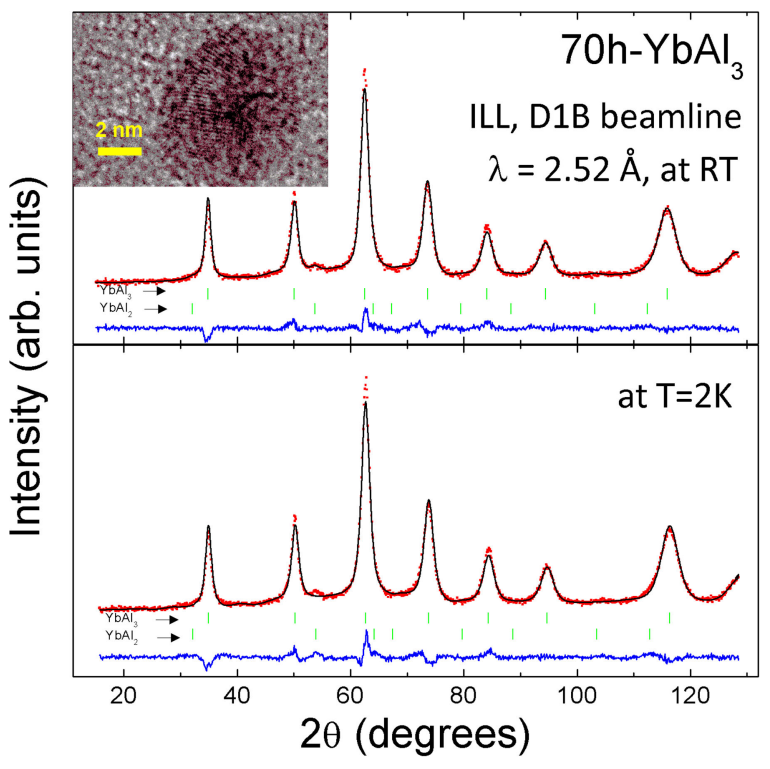

Fig. 2. Rietveld refinement of the ND pattern for $70 \mathrm{~h}-\mathrm{YbAl}_{3}$ (at RT and $T=2 \mathrm{~K}$ ). The theoretical calculation is depicted as a continuous (black) line and the experimental data as (red) points. The blue line below the spectra is the difference between the calculated and experimental patterns. Bragg reflections of the $\mathrm{YbAl}_{3}$ and $\mathrm{YbAl}_{2}$ phases are also depicted as green vertical lines. The left upper inset shows a TEM image of a $70 \mathrm{~h}-\mathrm{YbAl}_{3}$ nanoparticle.

reliability factors $\chi^{2}=2.56$ and $R_{B}=5.42$ (for the $\mathrm{YbAl}_{3}$ phase). Traces of $\mathrm{YbAl}_{2}$ were also found in the patterns, accounting for less than $1 \mathrm{wt} \%$, as obtained after Rietveld refinements. These values are similar to those obtained in other $\mathrm{YbAl}_{3}$ milled alloys [EchevarriaBonet 2018] and in the same order of magnitude of those found by TEM results. Indeed, TEM, shown in the inset of Fig. 2, confirms the order of magnitude of the mean particle size.

Structural analysis was completed via ND at different temperatures in the $70 \mathrm{~h}-\mathrm{YbAl}_{3}$ nanometric sample. No changes (apart from the expected displacement of the Bragg peaks due to temperature variation) were detected in the patterns (see Fig. 2). The value of the lattice parameter at RT is $a=4.2028$ (1) $\AA$, in excellent agreement with previous 


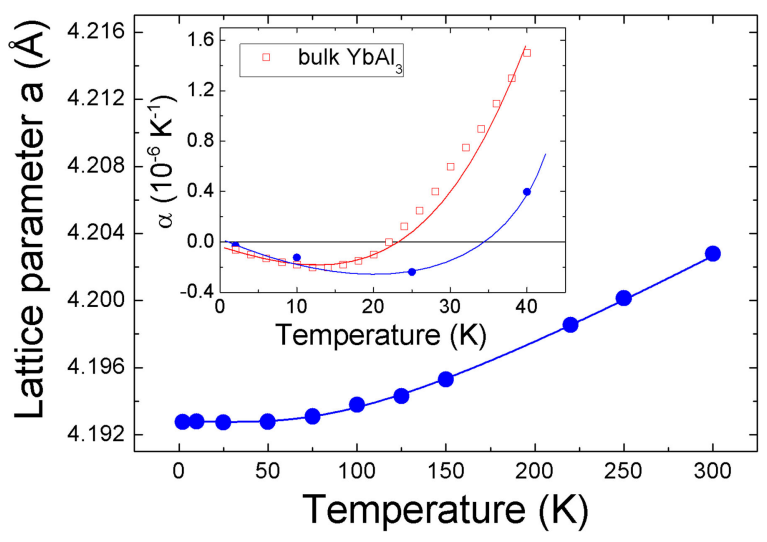

Fig. 3. Temperature-dependent lattice parameter for the $70 \mathrm{~h}-\mathrm{YbAl}_{3}$ sample, as obtained by Rietveld refinements. The experimental points are fitted to (2). Thermal expansion dependence with temperature, as obtained from (1) is shown in the inset and compared to that of bulk $\mathrm{YbAl}_{3}$. Lines in the inset are just guides for the eye.

results in this report and with Palenzona [1972]. This value decreases down to $a=4.1926(1) \AA$ at $T=2 \mathrm{~K}$. A small percentage of $\mathrm{YbAl}_{2}$ is also apparent in ND patterns, obtaining approximately 2.5(2) wt $\%$ at both temperatures. Reliability factors of $\chi^{2}=1.51$ and $R_{B}=3.37$ (for the $\mathrm{YbAl}_{3}$ phase) for the pattern at RT and $\chi^{2}=1.49$ and $R_{B}=1.8$ (for the $\mathrm{YbAl}_{3}$ phase) for the pattern at $2 \mathrm{~K}$ were obtained. The value of the particle size was $D=10(1) \mathrm{nm}$, consistent with SR-PXD analysis.

A thermal expansion study was performed using ND patterns, with temperatures from RT down to $2 \mathrm{~K}$. A cyclic refinement was performed for the different temperatures also with FullProf Suite, and the resulting values of the lattice parameter at these temperatures are shown in Fig. 3. The behavior of the lattice parameter is linear at high temperatures $(T>120 \mathrm{~K})$, providing a value of the thermal expansion coefficient of $\alpha=11.4(2) \times 10^{-6} \mathrm{~K}^{-1}$, obtained from $\alpha=1 / a(\Delta a / \Delta T)_{P}$. This value lies in the order of magnitude of bulk $\mathrm{YbAl}_{3}\left(17.0 \times 10^{-6} \mathrm{~K}^{-1}\right)$ [Iandelli 1972] even though it is slightly lower in the nanostructured $\mathrm{YbAl}_{3}$. However, it has to be taken into account that the temperature range was not the same as ours (between $25^{\circ} \mathrm{C}$ and $600{ }^{\circ} \mathrm{C}$ in Iandelli [1972]). On the other hand, if the thermal expansion is treated now for temperatures below $120 \mathrm{~K}$ as

$$
\alpha=\left(\frac{\partial \ln a}{\partial T}\right)_{P}
$$

we can plot the $\alpha(T)$ curve. The behavior of the thermal expansion with temperature in the nanostructured $\mathrm{YbAl}_{3}$ resembles that of a $\mathrm{YbAl}_{3}$ single crystal, where the curve has a minimum at low temperatures ( $T_{\min } \approx 14 \mathrm{~K}$ [Bud'ko 2008]). However, in the nano structured $\mathrm{YbAl}_{3}$, the minimum is more pronounced and $T_{\min }$ is shifted to higher temperatures $\left(T_{\min } \approx 25 \mathrm{~K}\right)$.

Moreover, a limited region of NTE $\alpha(T)<0$ is found around this $T_{\min }$, as it is the case in single crystals. However, the temperature region of NTE is larger in the $70 \mathrm{~h}-\mathrm{YbAl}_{3}$ (below $35 \mathrm{~K}$ ) than in the bulk (below $20 \mathrm{~K}$ ). NTE is also usually related to valence fluctuations [Yamada 2014] as it has already been reported in this compound. The $\mathrm{Yb}$ valence is 2.8 at $200 \mathrm{~K}$ and decreases to 2.65 as the temperature is reduced in $\mathrm{YbAl}_{3}$ single crystals [Suga 2005]; when being milled, a reduction of the $\mathrm{Yb}$ valence from 2.86 (in bulk) to 2.70 in nanostructured $\mathrm{YbAl}_{3}$ was determined from analysis of X-ray absorption near edge structure (XANES) spectra in Rojas [2008]. This behavior has been also reported in other mixed valence rare earth compounds, such as $\mathrm{YbAlB}_{4}$ [Matsumoto 2017] or $\mathrm{Sm}_{2.75} \mathrm{C}_{60}$ [Arvanitidis 2003].

We will use here the Debye-Grüneisen approximation as a good starting point for describing the contribution of the lattice to the thermal expansion of solid crystals. In this case, we will consider the anharmonic terms as negligible. According to Grüneisen's empirical law, the coefficient of thermal expansion $\alpha$, the isothermal compressibility $K$, and the specific heat at constant volume $C_{V}$ (including all contributions) are related as

$$
K=\frac{1}{V}\left(\frac{\Delta V}{\Delta P}\right)_{T} \quad \alpha=\gamma \frac{C_{V} K}{V}
$$

where $\gamma$ is the Grüneisen parameter. An estimation of the isothermal compressibility parameter $K$ may be determined for the bulk alloy from Kumar [2008], where a systematic study of high-resolution synchrotron X-ray diffraction under pressure was performed. The cell volume at $P=40 \mathrm{GPa}$ was reduced to $0.76 V_{0}$, with $V_{0}$ the cell volume at ambient pressure, thus obtaining $K=6.00(1) \times 10^{-12} \mathrm{~Pa}^{-1}$.

Using the Debye's elementary model for the lattice specific heat, we can write an expression for the temperature dependence of the lattice parameter (see Sayetat [1998]). For a cubic compound

$$
a=a_{0} \cdot\left[1+I_{a} \cdot T \cdot \phi\left(\frac{\theta_{D}}{T}\right)\right]
$$

where $a_{0}$ is the lattice parameter extrapolated at $0 \mathrm{~K}$, and

$$
I_{a}=\frac{k_{B} K \gamma}{V_{0}} .
$$

$\varphi\left(\theta_{D} / T\right)$ corresponds to Thacher's approximation for an easier calculation of the Debye function at temperatures $T>\theta_{D} / 10$ [Thacher 1960], as

$$
\phi\left(\frac{\theta_{D}}{T}\right)=\frac{\text { Poly_} N}{\text { Poly_} D}+\varepsilon
$$

with Poly_ $N$ and Poly_ $D$ being two polynomial functions of fourth order, whose coefficients are those indicated in Sayetat [1998], all of them dependent of the Debye temperature $\theta_{D}$. Moreover, $\varepsilon$ should be relatively small.

For the fitting procedure, we included only those temperatures higher than $25 \mathrm{~K}$ (approximately $T>\theta_{D} / 10$ ) [Sa 2012]. The curve $a(T)$ for the $70 \mathrm{~h}-\mathrm{YbAl}_{3}$ sample is fitted to (2) in Fig. 3, obtaining the following parameters: $\theta_{D}=440(70) \mathrm{K}, I_{a}=1.42(9) \times 10^{-5} \mathrm{~K}^{-1}$, and $a_{0}=4.1927(2) \AA$. From these values (and that of the isothermal compressibility $K$ ), we can also [by (3)] provide an estimate of the Grüneisen parameter of $\gamma=12.6(8)$, a value which is larger than that of metals, but similar to other SCES [Gegenwart 2016].

\section{B. Magnetic Characterization}

Fig. 4 shows the temperature dependence of the magnetization for both bulk (data taken from the literature [Rojas 2008]) and 70h- $\mathrm{YbAl}_{3}$ for comparison. Two main features are visible: (i) a broad maximum whose center is slightly shifted to higher temperatures for the milled alloy $\left(T_{\max }=125 \mathrm{~K}\right.$ for bulk [Bauer 2004], whereas $T_{\max }=133 \mathrm{~K}$ for $70 \mathrm{~h}-\mathrm{YbAl}_{3}$ ) and (ii) a plateau of the magnetization curve at low temperatures (around $20 \mathrm{~K}$ ) followed by an upturn for $T<20 \mathrm{~K}$. This broad maximum is usually associated with a Kondo behavior. A Kondo temperature $T_{K}$ of the order of $600 \mathrm{~K}$ has been found for $\mathrm{YbAl}_{3}$ bulk 


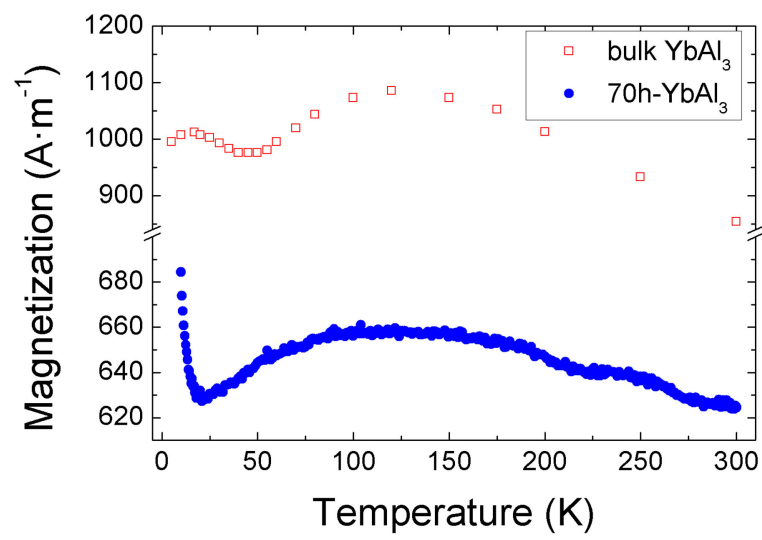

Fig. 4. Temperature-dependent magnetization curves for the bulk (data from [Rojas 2008]) and the $70 \mathrm{~h}-\mathrm{YbAl}_{3}$ milled alloy. A nonnegligible contribution of $\mathrm{YbAl}_{2}$ is visible at around $250 \mathrm{~K}$, as found in diffraction experiments.

[Bud'ko 2008]. If we scale the $T_{\max }$ with this Kondo temperature for both bulk and milled alloy, an approximate value of $T_{K} \approx 650 \mathrm{~K}$ can be estimated for the $70 \mathrm{~h}-\mathrm{YbAl}_{3}$ compound. In order to provide a more accurate value of $T_{K}$, quasielastic neutron scattering experiments should be performed. This result is in good agreement with the increase of the Debye temperature found in the thermal expansion behavior above that also indicated a $T_{K}$ enhancement, modifying the coherence effects at low temperatures, as reported in previous results of milled $\mathrm{YbAl}_{3}$ alloys [Echevarria-Bonet 2018]. The behavior of the curves here is in good agreement with those in the literature [Rojas 2008] for milled $\mathrm{YbAl}_{3}$ alloys though $T_{\max }$ values are slightly different. Ohara [2010] noted, in $\mathrm{YbAl}_{3}$ single crystals, not only a subtle shift of the $T_{\max }$ to lower temperatures but also an enhancement of the magnetic susceptibility with increasing pressure (1.07 GPa) [Ohara 2010]. Somehow, this could indicate a negative pressurelike effect when $\mathrm{YbAl}_{3}$ is nanostructured. This same effect occurs in $\mathrm{Yb}_{1-x} \mathrm{Lu}_{x} \mathrm{Al}_{3}$, in which the $T_{\max }$ shifted to higher temperatures with increasing Lu concentration [Bauer 2004], leading to the idea that nanostructuring this alloy is similar to applying negative pressure or chemical tuning. Not only the $T_{\max }$ shifts to higher temperatures but also the value of the magnetic susceptibility decreases with milling, as it does with higher Lu concentrations [Bauer 2004]. This decrease of the magnetic susceptibility is a significant sign of the increasing presence of $\mathrm{Yb}^{2+}$ contribution (nonmagnetic) at high temperatures [Rojas 2008]. If we take into account the Coqblin-Schriefer model, $\chi(0) \propto 1 / T_{K}$ [Rajan 1983], the decrease of the magnitude of the magnetic susceptibility would lead to an increase of the Kondo temperature, consistently with thermal expansion results and the position of $T_{\max }$ in the magnetic susceptibility (see Fig. 4). This is also true in other Yb-based compounds, such as in the $\mathrm{Yb}_{1-x} \mathrm{Y}_{x} \mathrm{CuAl}$ series [Rojas 2018].

Regarding (ii), the upturn of the magnetization (or susceptibility) at low temperatures may be related to the presence of magnetic impurities or to the change of degrees of freedom. The latter can be understood as a modification of the structure of the alloys when nanostructuring, leading to the increase of the $\mathrm{Yb}^{3+}$ contribution on the surface of the particles at low temperatures. Signs of valence change with temperatures are evident in this analysis. It would be of interest to follow the valence fluctuations with temperatures for milled alloys.

\section{CONCLUSION}

In conclusion, we have shown that the reduction of size $(\sim 11 \mathrm{~nm})$ in SCES $\mathrm{YbAl}_{3}$ has not only electronic effects in the Kondo temperature (from $600 \mathrm{~K}$ in bulk to approximately $650 \mathrm{~K}$ in nanostructured material) and changes in the coherence effects and in the low-temperature FL behavior, but also has important consequences in the lattice dynamics, because the region of the NTE is slightly larger (below $40 \mathrm{~K}$ ) when compared to that of the bulk (below $20 \mathrm{~K}$ ). Magnetization results at low temperatures are intriguing and additional experiments are needed to further elucidate the origin of the upturn at low temperatures.

\section{ACKNOWLEDGMENT}

This work was supported in part by the Spanish Ministerio de Ciencia, Innovación y Universidades under Project MAT2017-83631-C3-3-R and in part by the Principado de Asturias, Spain, under Project GRUPINN IDI/2018/000185. The work of C. EchevarriaBonet was supported by Banco Santander for their Mobility grant for professors and researchers from University of Oviedo (2019)

\section{REFERENCES}

Álvarez-Alonso P, Gorria P, Blanco J A, Sánchez-Marcos J, Cuello G J, Puente-Orench I, Rodríguez-Velamazán J A, Garbarino G, de Pedro I, Rodríguez Fernández J, Sánchez Llamazares J L (2011), "Magnetovolume and magnetocaloric effects in $\mathrm{Er}_{2} \mathrm{Fe}_{17}$," Phys. Rev. B, vol. 86, 184411, doi: 10.1103/PhysRevB.86.184411.

Arvanitidis J, Papagelis K, Margadonna S, Prassides K, Fitch A N (2003), “Temperatureinduced valence transition and associated lattice collapse in samarium fulleride," Nature, vol. 425, pp. 599-602, doi: 10.1038/nature01994.

Attfield J P (2018), "Mechanisms and materials for NTE," Frontiers Chem., vol. 6, 371, doi: $10.3389 /$ fchem.2018.00371.

Bauer E D, Booth C H, Lawrence J M, Hundley M F, Sarrao J L, Thompson J D, Riseborough P S, Ebihara T (2004), "Anderson lattice behavior in $\mathrm{Yb}_{1-x} \mathrm{Lu}_{x} \mathrm{Al}_{3}$," Phys. Rev. B, vol. 69, 125102, doi: 10.1103/PhysRevB.69.125102.

Bud'ko S L, Frederick J C, Mun E D, Canfield P C, Schmiedeshof G M (2008), "Thermal expansion, heat capacity and magnetostriction of $\mathrm{RAl}_{3}(\mathrm{R}=\mathrm{Tm}$, Yb, Lu) single crystals," J. Phys.: Condens. Matter, vol. 20, 025220, doi: 10.1088/0953-8984/20/02/025220.

Buschow K H J, Grössinger R (1987), "Spontaneous volume magnetostriction in $\mathrm{R}_{2} \mathrm{Fe}_{14} \mathrm{~B}$ compounds," J. Less-Common Metals, vol. 135, pp. 39-46, doi: 10.1016/0022-5088(87)90336-5.

Chatterjee S, Ruf J P, Wei H I, Finkelstein K D, Schlom D G, Shen K M (2017), "Lifshitz transition from valence fluctuations in $\mathrm{YbAl}_{3}$," Nature Commun., vol. 8, 852, doi 10.1038/s41467-017-00946-1.

Ebihara T, Bauer E D, Cornelius A L, Lawrence J M, Harrison N, Thompson J D, Sarrao J L, Hundley M F, Uji S (2003), "Dependence of the effective masses in $\mathrm{YbAl}_{3}$ on magnetic field and disorder," Phys. Rev. Lett., vol. 90, 166404, doi: 10.1103/PhysRevLett.90.166404

Echevarria-Bonet C (2014), "Size induced electronic and magnetic changes in nanometric rare earth alloys," Ph.D. dissertation, Univ. Cantabria, Santander, Spain.

Echevarria-Bonet C, Rojas D P, Espeso J I, Rodríguez Fernández J, Rodríguez Fernández L, Bauer E, Burdin S, Magalhães S G, Fernández Barquín L (2018), "Breakdown of the coherence effects and Fermi liquid behavior in $\mathrm{YbAl}_{3}$ nanoparticles," J. Phys., Condens. Matter, vol. 30, 135604, doi: 10.1088/1361-648X/aab0c7.

Gegenwart P (2016), "Grüneisen parameter studies on heavy fermion quantum criticality," Rep. Prog. Phys., vol. 79, 114502, doi: 10.1088/0034-4885/79/11/114502.

Görlach T, Pfleiderer C, Grube K, Löhneysen H V (2005), "Low-temperature properties of $\mathrm{YbAl}_{2}$," Phys. Rev. B, vol. 71, 033101, doi: 10.1103/PhysRevB.71.033101.

Guillaume C E (1897), "Recherches sur les aciers au nickel. Dilatations aux temperatures elevees; resistance electrique," Compt. Rend. Acad. Sci., vol. 125, pp. 235-238. [Online]. Available: https://gallica.bnf.fr/ark:/12148/bpt6k30813/f235.image

Hiess A, Boucherle J X, Givord F, Schweizer J, Lelièvre-Berna E, Tasset F, Gillon $\mathrm{B}$, Canfield P C (2000), "Magnetism in intermediate-valence $\mathrm{YbAl}_{3}$ : A polarized neutron diffraction study," J. Phys.: Condens. Matter, vol. 12, pp. 829-840, doi: 10.1088/0953-8984/12/6/307.

Iandelli A, Palenzona A (1972), "Magnetic susceptibility and expansion coefficient of the intermetallic compounds $\mathrm{YbAl}_{2}$ and $\mathrm{YbAl}_{3}$, , J. Less Common Metals, vol. 29, pp. 293-297, doi: 10.1016/0022-5088(72)90117-8. 
Kumar R S, Svane A, Vaitheeswaran G, Kanchana V, Bauer E D, Hu M, Nicol M F, Cornelius A L (2008), "Pressure-induced valence change in $\mathrm{YbAl}_{3}$ : A combined high-pressure inelastic x-ray scattering and theoretical investigation," Phys. Rev. B, vol. 78, 075117, doi: 10.1103/PhysRevB.78.075117.

Li W-H, Wu S Y, Yang C C, Lai S K, Lee K C, Huang H L, Yang H D (2002), "Thermal contraction of Au nanoparticles," Phys. Rev. Lett. vol. 89, 135504, doi: 10.1103/PhysRevLett.89.135504.

Matsumoto Y, Kuga K, Tomita T, Küchler R, Nakatsuji S (2017), "Anisotropic thermal expansion of $\alpha$-YbAlB 4 ," J. Phys., Conf. Ser., vol. 807, 022005, doi: $10.1088 / 1742-6596 / 807 / 2 / 022005$.

Moriarty J L, Humphreys J E, Gordon R O, Baenziger N C (1966), "X-ray examination of some rare-earth-containing binary alloy systems," Acta Crystallographica, vol. 21, pp. 840-841, doi: 10.1107/S0365110X6600402X.

Ohara S, Adachi Y, Yamashita T, Sakamoto I (2010), "Pressure effects on magnetic susceptibility of intermediate-valence compound $\mathrm{YbAl}_{3}$," J. Phys.: Conf. Ser., vol. 200, 012150, doi: 10.1088/1742-6596/200/1/012150.

Palenzona A (1972), "The ytterbium-aluminum system," J. Less Common Metals, vol. 29, pp. 289-292, doi: 10.1016/0022-5088(72)90116-6.

Rajan V T (1983), "Magnetic susceptibility and specific heat of the Coqblin-Schrieffer model," Phys. Rev. Lett., vol. 51, pp. 308-311, doi: 10.1103/PhysRevLett.51.308.

Rodríguez-Carvajal J (1993), "Recent advances in magnetic structure determination by neutron powder diffraction," Phys. B: Condens. Matter, vol. 192, pp. 55-69, doi: 10.1016/0921-4526(93)90108-I.

Rojas D P, Fernández Barquín L, Espeso J I, Rodríguez Fernández J, Chaboy J (2008), "Reduction of the $\mathrm{Yb}$ valence in $\mathrm{YbAl}_{3}$ nanoparticles," Phys. Rev. B, vol. 78, 094412, doi: 10.1103/PhysRevB.78.094412.

Rojas D P, Gandra D G, Medina A N, Fernández Barquín L, Gómez Sal J C (2018), "Kondo temperature and heavy fermion behavior in $\mathrm{Yb}_{1-\mathrm{x}} \mathrm{Y}_{\mathrm{x}} \mathrm{CuAl}$ series of alloys," Phys. B: Condens. Matter, vol. 536, pp. 176-181, doi: 10.1016/j.physb.2017.09.096.
Rossi L, Bobel A, Wiedmann S, Küchler R, Motome Y, Penc K, Shannon N, Ueda H, Bryant B (2019), "Negative thermal expansion in the plateau state of a magnetically frustrated spinel," Phys. Rev. Lett., vol. 123, 027205, doi: 10.1103/PhysRevLett.123.027205.

Rowe D M, Kuznetsov V L, Kuznetsova L A, Min G (2002), "Electrical and thermal transport properties of intermediate valence $\mathrm{YbAl}_{3}$," J. Phys. D: Appl. Phys., vol. 35, pp. 2183-2186, doi: 10.1088/0022-3727/35/17/315.

Sa B, Zhou J, Sun Z (2012), "First-principles investigation of mechanical and thermodynamic properties of the rare earth intermetallic $\mathrm{YbAl}_{3}$ under pressure," Intermetallics, vol. 22, pp. 92-98, doi: 10.1016/j.intermet.2011.11.005.

Sayetat F, Fertey P, Kessler M (1998), "An easy method for the determination of Debye temperature from thermal expansion analyses," J. Appl. Crystallogr., vol. 31, pp. 121127, doi: $10.1107 /$ S0021889897006936.

Suga S, Sekiyama A, Imada S, Shigemoto A, Yamasaki A, Tsunekawa M, Dallera C, Braicovich L, Lee T-L, Sakai O, Ebihara T, Ōnuki Y (2005), "Kondo lattice effects of $\mathrm{YbAl}_{3}$ suggested by temperature dependence of high-accuracy highenergy photoelectron spectroscopy," J. Phys. Soc. Jpn., vol. 74, pp. 2880-2884, doi: 10.1143/JPSJ.74.2880.

Thacher H C (1960), "Rational approximations for the Debye functions," J. Chem. Phys., vol. 32, 638, doi: 10.1063/1.1730772.

van Daal H J, van Aken P B, Buschow K H J (1974), "The Seebeck coefficient of $\mathrm{YbAl}_{2}$ and $\mathrm{YbAl}_{3}$," Phys. Lett. A, vol. 49, pp. 246-248, doi: 10.1016/0375-9601(74)90870-6.

Yamada I, Shiro K, Etani H, Marukawa S, Hayashi N, Mizumaki M, Kusano Y, Ueda S, Abe H, Irifune T (2014), "Valence transitions in negative thermal expansion material $\mathrm{SrCu}_{3} \mathrm{Fe}_{4} \mathrm{O}_{12}$," Inorg. Chem., vol. 53, pp. 10563-10569, doi: 10.1021/ic501665c.

Zheng X G, Kubozono H, Yamada H, Kato K, Ishiwata Y, Xu C N (2008), "Giant negative thermal expansion in magnetic nanocrystals," Nature Nanotechnol., vol. 3, pp. 724726, doi: 10.1038/nnano.2008.309. 